\title{
Uncertainty in loading and control of an active column critical to buckling
}

\author{
G.C. Enss ${ }^{\mathrm{a}, *}$, R. Platz ${ }^{\mathrm{b}}$ and H. Hanselka $\mathrm{a}^{\mathrm{a}, \mathrm{b}}$ \\ ${ }^{a}$ System Reliability and Machine Acoustics SzM, TU Darmstadt, Darmstadt, Germany \\ ${ }^{\mathrm{b}}$ Fraunhofer Institute for Structural Durability and System Reliability LBF, Darmstadt, Germany
}

\begin{abstract}
Buckling of load-carrying column structures is an important design constraint in light-weight structures as it may result in the collapse of an entire structure. When a column is loaded by an axial compressive load equal to its individual critical buckling load, a critically stable equilibrium occurs. When loaded above its critical buckling load, the passive column may buckle. If the actual loading during usage is not fully known, stability becomes highly uncertain.

This paper presents an approach to control uncertainty in a slender flat column structure critical to buckling by actively stabilising the structure. The active stabilisation is based on controlling the first buckling mode by controlled counteracting lateral forces. This results in a bearable axial compressive load which can be theoretically almost three times higher than the actual critical buckling load of the considered system. Finally, the sensitivity of the presented system will be discussed for the design of an appropriate controller for stabilising the active column.
\end{abstract}

Keywords: Uncertainty, buckling, active stabilisation

\section{Introduction}

Technical structures may be exposed to mechanical stress, thermal stress, chemical stress and many other stresses during their usage. Load-carrying technical structures are designed to withstand mechanical loads and may fail by overloads or fatigue. Whereas fatigue is generally a long-term failure, overloads may result in a sudden failure. An example for such sudden failure is buckling of axially compressed slender structures. If a slender column is loaded by a load above its critical buckling load, it may buckle. Eventually, this may lead to the collapse of an entire structure. A structure critical to buckling can be very sensitive to disturbances and other uncertainty. For this, a highly sensitive structure is used as an example in this paper to decscribe, assess and control uncertainty in the following and future work. The understanding of how uncertainty is propagated may later be transferred to other uncertain load-carrying structures.

This study is part of the Collaborative Research Centre (Sonderforschungsbereich SFB) 805: Control of Uncertainty in Load-Carrying Systems in Mechanical Engineering, which is publicly funded by the Deutsche Forschungsgemeinschaft (DFG). Within this workgroup, engineers and mathematicians have set up the following working hypothesis of uncertainty: "Uncertainty occurs when process properties of a system can not or only partially be determined" [1]. According to this working hypothesis, uncertainty may occur in a load-carrying system e.g. due to unforeseen overloads during processes in operation.

Stability of axially compressed columns has been studied thoroughly for four different boundary conditions by Euler [2]. A mathematical solution of the critical buckling load $P_{c r}$ of compressed columns and beam-columns also subjected to lateral forces has been treated in the monograph of Timoshenko and Gere [3]. To prevent buckling, standards and design guidelines propose the use of safety factors, e.g. [4]. However, safety factors may lead to

\footnotetext{
*Corresponding author: G.C. Enss, TU Darmstadt, System Reliability and Machine Acoustics SzM, Magdalenenstrasse 4, 64289 Darmstadt, Germany. E-mail: enss@szm.tu-darmstadt.de.
} 
additional weight of the load-carrying structure that is only utilised in few occasions when the structure is actually overloaded. For normal loading condition, the structure would be oversized. For increasing safety factors, passive modifications of the structure like increased geometrical moment of inertia would lead to more stability. To discuss the effect of passive solutions further, the analytical equation of the critical buckling load in a single column critical to buckling is well known,

$$
P_{c r}=\frac{\pi^{2} \cdot E I}{(0.7 L)^{2}},
$$

with bending stiffness $E I$ and effective buckling length 0.7 L, Fig. 1 and [3].

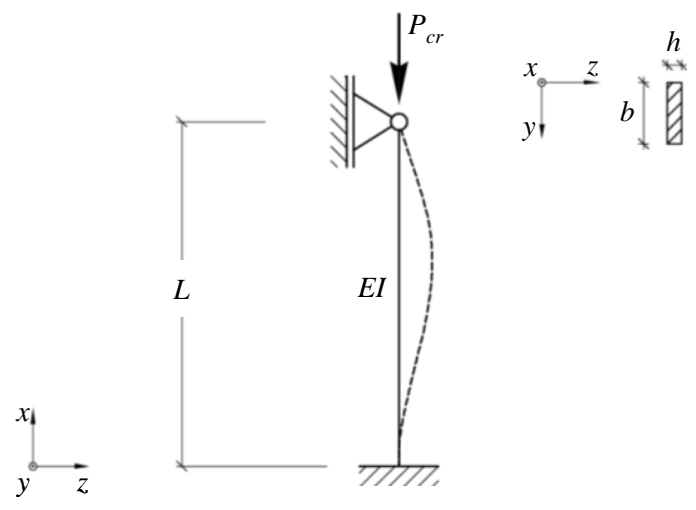

Fig. 1. Passive axially loaded column.

Potentials of passive strengthening against buckling will be discussed in the following. The buckling load depends upon material property Young's modulus $E$ and geometric properties length $L$ and moment of inertia $I$ with width $b$ and column thickness $h$ [3],

$$
I=\frac{b \cdot h^{3}}{12}
$$

The critical load $P_{c r}$ varies by simply varying the governing material and geometrical parameters $E, L$ and $h$. As an example, Fig. 2 shows the variation of $P_{c r}$ by its scaling factor due to scale variations in $E, L$ and $h$ between 0 and 2 .

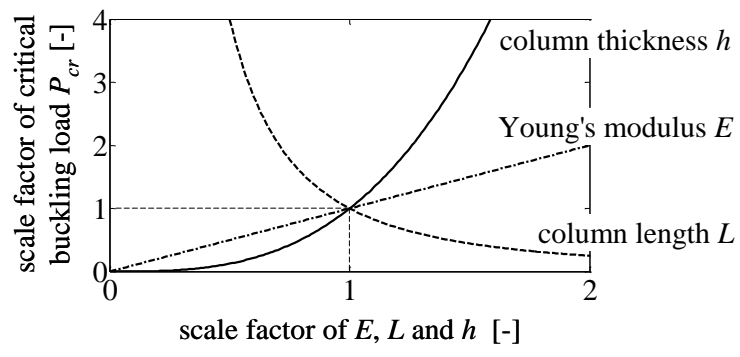

Fig. 2. Scaling of normalised critical buckling load $P_{c r}$ for variation of $E, L$ and $h$.

Of course, Young's modulus $E$ has a linear effect on the buckling load. Choosing the twofold value for $E$ also affects in a critical buckling load $P_{c r}$ twice as high. Furthermore, $P_{c r}$ has a reciprocal quadratic dependence on the length of the column $L$ in Eq. (1) and a cubic dependence on the column's thickness $h$ in Eq. (2). This means that a 
column of half the length would carry a fourfold critical buckling load and a column twice as thick would carry an eightfold critical buckling load.

In technical structures, though, the possibility of changing the length, thickness or even the material of a structure is often not given due to strict boundary conditions and system specifications. Additionally, if the structure is oversized by a passive safety factor to avoid buckling, the additional weight may always be carried by the system during its entire usage. To save weight, it would be more efficient to have only a slightly oversized structure that carries usually loads that are applied under normal and most frequent conditions. The structure should be stabilised just during the few occasions that the structure is overloaded. Therefore, a premature fatigue failure due to stabilising control forces is assumed to be negligible in comparison with the buckling failure at the first ocurrence of an overload during operation.

Generally, designing a load-carrying structure, assumptions are made to describe its actual loading e.g. expected load $\bar{P}$ and frequency distribution $H(P)$. Likewise, structural properties may also scatter, for example the expected critical buckling load $\bar{P}_{c r}$ with its frequency distribution $H\left(P_{c r}\right)$. Now, real structures under real loading conditions may fail when actual loading $P$ is greater than the critical load $P_{c r}$, which can be described by an overlap of the frequency distributions $H(P)$ and $H\left(P_{c r}\right)$, Fig. 3.

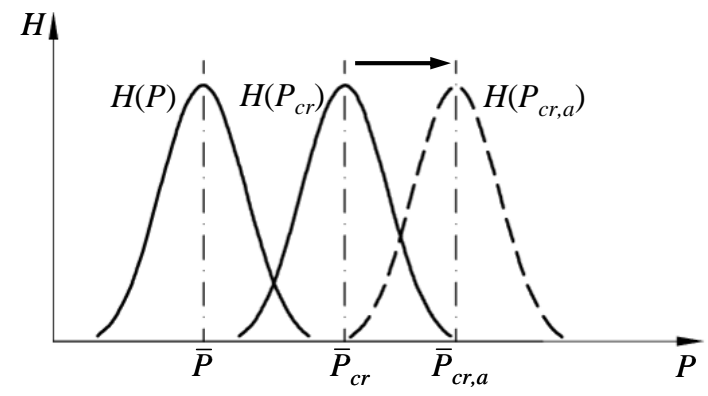

Fig. 3. Frequency distributions for passive (-) and active (---) slender column structure.

If it would be possible to stabilise the same slender structure actively to withstand an excessive but only short time overloading, the frequency distribution $H\left(P_{c r, a}\right)$ with the expected new and higher active value $\bar{P}_{c r, a}$ will shift to higher strength, Fig. 3. By raising the bearable critical load, the intersection between the frequency distributions $H(P)$ and $H\left(P_{c r, a}\right)$ would be smaller and the probability of failure would decrease, Fig. 3, dashed line. Thus, uncertainty that results from failures due to unknown loading during operation could be reduced. In this paper, a column as described above and critical to buckling under axial compressive load $P$ and actively stabilised for few occasions only will be examined.

In literature, several approaches to stabilise column structures actively using smart materials are present. They are mostly based on applying additional forces or bending moments on the column's surface or in the clamped or pinned supports [5-8]. Those works are based on shape control with bending moments along the length of the column, using piezoelectric patch actuators attached to the column's surface only. The use of piezoelectric patch actuators is, however, limited because of its inherent characteristics, such as small available strain of $0.1-0.2 \%$ [9]. However, an increase of the critical buckling load up to a factor of 5.6 was achieved experimentally for a thin pinned-pinned beam with attached piezoelectric patch actuators along the entire length of the beam and gap stiffeners between the actuators [5]. Due to the small beam thickness, control forces were also small. Another concept achieved a factor of $8.8 P_{c r}$ by controlling the first two buckling modes in numerical simulations also using piezoelectric patch actuators along the pinned-pinned beam's length [8].

In other works, concepts are presented applying bending moments through embedded shape memory alloys (SMA) in a predeflected composite column [10] and experimentally validated with an increase of the buckling load of $11 \%$. Comparing to piezoelectric actuators, SMA may apply large deflections at the expense of applicable forces and operating frequency. Therefore, their field of application is limited for one single stabilisation or static stabilisation of predeflected structures, as shown in [10]. 
Other approaches apply lateral forces onto the column. One approach was made with a bracing of two electromagnets applying deflection proportional forces onto the column, resulting in a stabilisation of the first buckling mode [11].

In this paper, the examined column has to withstand axial compressive loads as well as uncertain lateral disturbance forces. Those may occur during an actual operation and may have considerable influence on the system's stability and buckling behaviour. Allocating actuators along the column's length and surface are not taken into account since they would be exposed to the environment. In addition, the exposed actuators along the surface might also restrict the correct function of a plain and clean surface, e.g. the surface of a piston rod in a hydraulic cylinder needs to pass the sealings without irregularities on the surface. Therefore and in this work, the active stabilisation will be restricted to a discrete lateral active force $F_{a}$, which is located not in but close to the column's clamped base.

\section{Description of investigated active column system}

The considered active system is a flat slender column with a rectangular cross section $b \cdot h$, pinned at the upper end $x=L$ and clamped at the base $x=0$, Fig. 4. Its properties are: length $L$, bending stiffness $E I$, density $\rho$ and modal damping $\theta$.

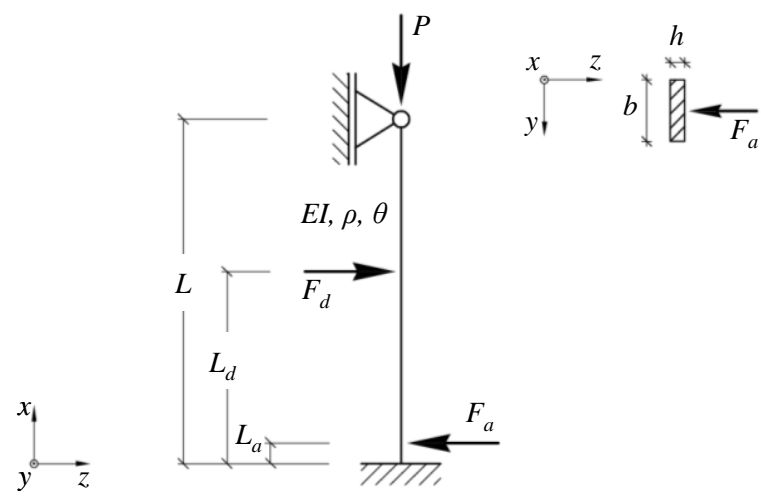

Fig. 4. Column system with disturbance force $F_{d}$ and active force $F_{a}$.

The column is loaded axially by $P$ almost as high as the critical buckling load $P_{c r}$, (1) with $P \approx 0.9 P_{c r}$. Due to its rectangular cross section it buckles preferably in $x$-z-plane after an impact disturbance force $F_{d}$ is applied at $x=L_{d}$. A controlled active force $F_{a}$ may act close to the column's base $x=L_{a}=L / 30$ if the column needs to be stabilised quickly due to sudden but short-term overloading. The reason for choosing position $L_{a}$ of the active force is to keep most of the surface of the column free from additional actuators. At $L_{a}$, column deflections are small and active control forces may become relatively high favouring the use of piezoelectric stack actuators for active stabilisation. This location is reasonable for the chosen restrictions and boundary conditions. The properties of the column system presented in Fig. 4 are summarised in Table 1.

Table 1

Properties of considered column system

\begin{tabular}{ccc}
\hline Geometrical properties & Material properties (AlMg3) & Boundary conditions / Mechanical properties \\
\hline$L=300 \mathrm{~mm}$ & $E=70 \mathrm{kN} / \mathrm{mm}^{2}$ & pinned end at $x=L$ \\
$b=20 \mathrm{~mm}$ & $\rho=2660 \mathrm{~kg} / \mathrm{m}^{3}$ & clamped base at $x=0$ \\
$h=1 \mathrm{~mm}$ & $\theta=1 \%$ & $P$ constant at $x=L, x$-direction \\
$L_{d}=168 \mathrm{~mm}$ & & $F_{d}$ pulse at $x=L_{d}, z$-direction \\
$L_{a}=10 \mathrm{~mm}$ & $F_{a}$ controlled force at $x=L_{a}, z$-direction \\
\hline
\end{tabular}


For the given properties, (1) yields the individual buckling load $P_{c r}=26.14 \mathrm{~N}$. This value is calculated considering only geometric and material properties and neglecting damping, possible disturbances $F_{d}$ and the dynamic behaviour of the column structure.

\section{Concept of active stabilisation}

Generally, a passive structure should carry the loads it is designed for at all times. In case of the proposed active structure, active stabilisation is intended to be activated to prevent buckling failure only if an overload or critical deflection is detected. In previous works [5-8,12], the concept of active stabilisation was to force the active column into its static second or higher bending deflection shape. In this work, however, the concept of active stabilisation is based on a similar concept, but the way to force the column into its second mode shape is different. The moments stabilising the column are not distributed along the column's length as in the cited works but there is just one moment generated by active forces acting close to the fixed end. For an ideal system, forcing the column into its second bending deflection shape results in a reduction of the buckling length $L$ to effective buckling length $L_{d} \approx 0.6 L,[12]$. The critical load $P_{c r}$ increases by factor 2.9 for the given boundary conditions theoretically because of the quadratic relationship of the buckling length $L$ in Eq. (1), [3].

The approach for active stabilisation of an axially compressed column is based on the linear superposition of deflection shapes caused by an assumed disturbing force $F_{d}$, exposed at the most critical location $L_{d}$ and leading to the first buckling mode and the counteracting force $F_{a}$, Fig. 5, [12]. The deflection shape $w_{d}$ from disturbance force $F_{d}$ prior to large buckling deflection is superimposed by the deflection shape $w_{a}$ caused by the counteracting active force $F_{a}$ in the opposite direction, resulting in the approximate second bending deflection shape, Fig. 5.

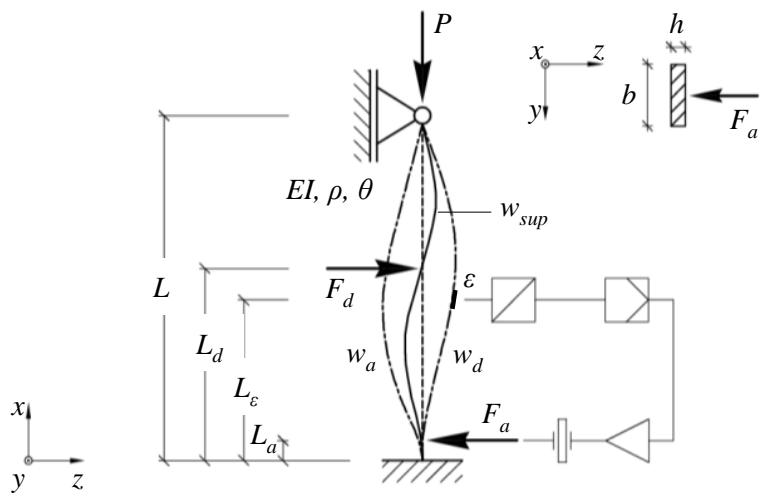

Fig. 5. Actively controlled column system, approach in one direction.

The deflection $w_{d}$ resulting from $F_{d}$ is detected by strain gauge sensors that measure strain $\varepsilon$ on the column's surface at $x=L_{\varepsilon}$, Fig. 5. The measured signal is then processed in a signal based PID feedback control algorithm. The controller output is amplified to drive a piezoelectric stack actuator. This actuator applies the active force $F_{a}$ to the column near its fixed end $x=L_{a}$ which causes a deflection of $w_{a}$ on the column. The continuous superimposed deflection line $w_{\text {sup }}$, Fig. 5, results from superimposing both deflection lines $w_{d}$ and $w_{a}$. First experimental studies lead to a $15 \%$ increase of the carried load $P$ compared to the experimentally tested critical buckling load $P_{c r}$, [12], see Section 4.

The major advantage with this active approach is to stabilise the column without bonding piezoelectric patch actuators on the column's surface but to apply one piezoelectric stack actuator close to the column's base, Fig. 9a. Through the implementation of a rather simple and fast control algorithm, the column could be stabilised in real-time as well as withstand lateral disturbance forces $F_{a}$ which were not analysed thoroughly in previous works [5-8]. In comparison to other works, e.g. [10], no predeflected column is stabilised, but the straight active column system reacts in real-time due to deflections caused by the disturbance force which is applied manually. 
However, the presented concept also has drawbacks in terms of limited controllability of the system. By being able to apply the active force in negative $z$-direction only, the system easily buckles in the same negative $z$-direction when the active force is applied too abruptly. Furthermore, the resulting superimposed deflection shape is only an approximated second bending deflection shape. This shape can only be realised as long as both $F_{d}$ and $F_{a}$ are applied at the same time.

Due to these drawbacks, a new concept was developed with the same restrictions for the active force and for a stabilisation in both positive and negative $z$-direction using a second piezoelectric stack actuator to generate the active force, Figs 6 and $9 \mathrm{~b}$.

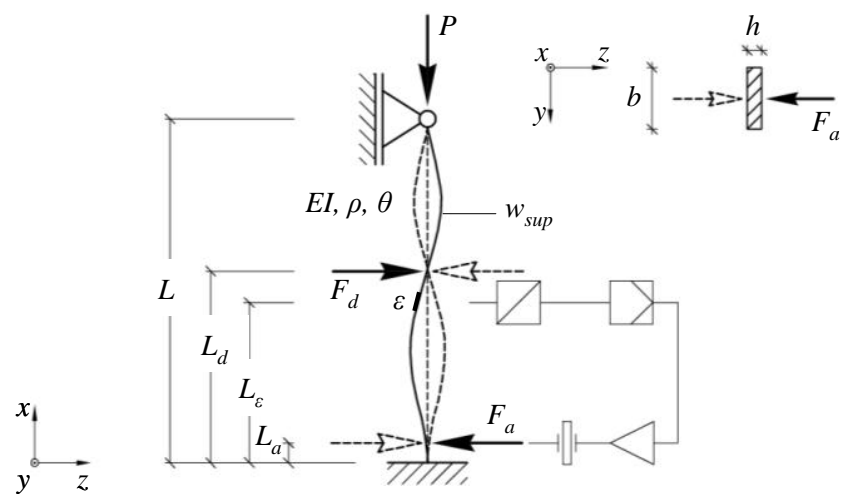

Fig. 6. Actively controlled column system, approach in two directions.

By forcing the column into its second bending deflection shape, the deflection at the nodal point $x=L_{d}$ stays zero for all times which is equivalent to controlling the first buckling mode. Figure 7 shows the feedback control loop for the designed system. When the column is disturbed by a disturbance force $F_{d}$, the column deflects slightly which is detected by the strain gauge sensor and is processed as voltage $U_{\varepsilon}$. The signal is then controlled by a signal based feedback controller to generate the control signal $U_{a}$ which drives the piezo amplifiers and the two piezoelectric stack actuators. The actuators then apply a stabilising active force $F_{a}$ to stabilise the column. For a setpoint of $\varepsilon=0$, the nodal point at $L_{d}$ has zero deflection which can be interpreted as a virtual pinned support, leading to a higher critical buckling load.

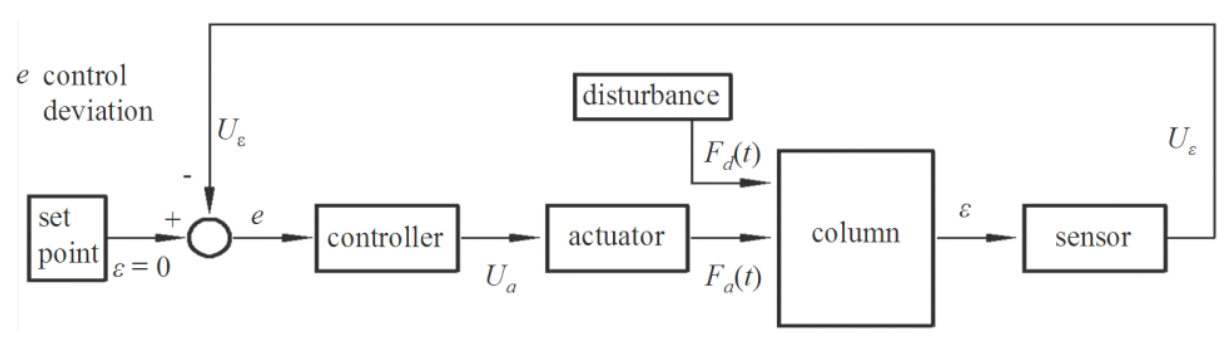

Fig. 7. Feedback control loop.

By changing the axial load $P$, the system's behaviour changes inherently. Naturally, eigenfrequencies depend on the axial load $P$, Fig. 8. Generally, the higher the axial compressive load $P$ becomes, the lower are the eigenfrequencies. In Fig. 8, the first eigenfrequency $f_{1}$ becomes zero for an axial compressive load of $P=P_{c r}$, which means that the column is not capable of vibrating in its first bending mode shape anymore. The first eigenfrequency becomes zero, when the stiffness of the column becomes zero and, thus, the column may buckle.

A further increase of the axial load leads to an unstable column system which may be controlled by time-dependent active force $F_{a}(t)$. When its first buckling mode is controlled, the column is still capable of vibrating in the second eigenfrequency of about $f_{2}=105.7 \mathrm{~Hz}$ in its second bending mode shape. With a rising axial com- 


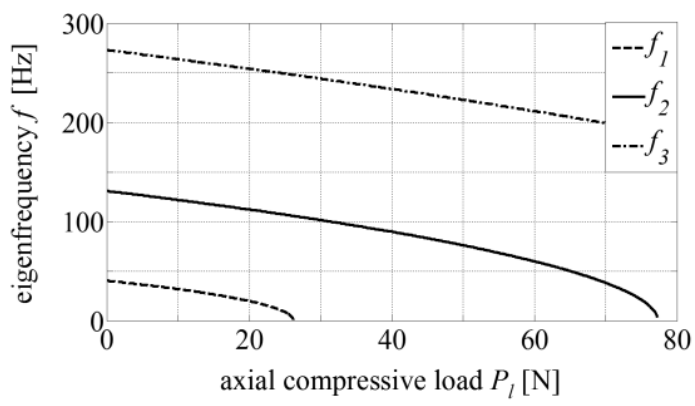

Fig. 8. First three eigenfrequencies of axially compressed column.
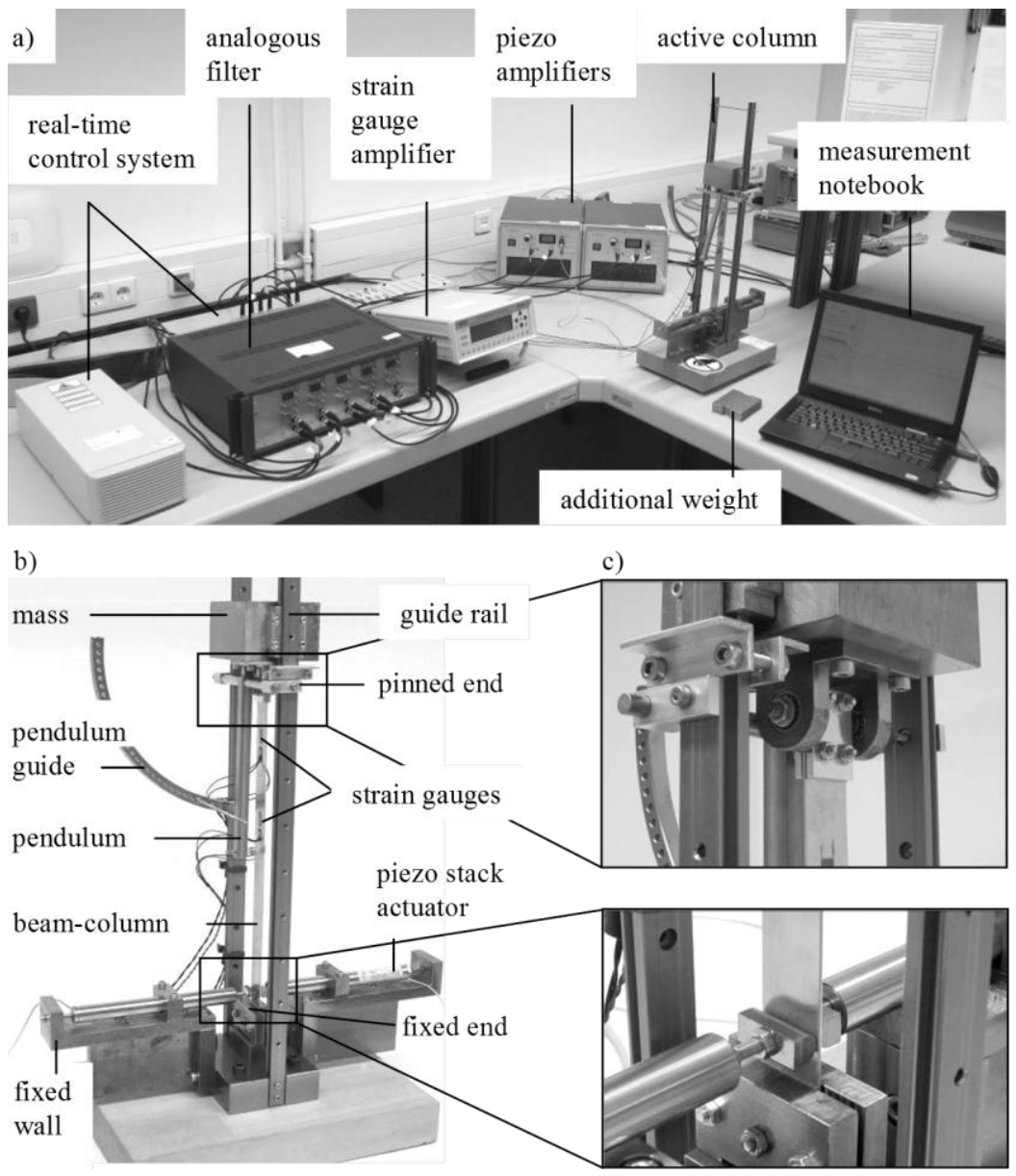

Fig. 9. Active column system (a) overview of the demonstrator with all instrumentation, (b) demonstrator for buckling control in two directions, (c) detail picture of pinned support with limit stop device (top) and fixed end with active force generated by two piezoelectric stack actuators (bottom).

pressive load, $f_{2}$ decreases with a gradient of $-1 \mathrm{~Hz} / \mathrm{N}$ at $P=P_{c r}$. With the structure deflected in its second bending deflection shape, the column is stabilised and may carry almost three times the critical buckling load, theoretically, as long as the deflection at the nodal point $x=L_{d}$ stays always zero.

Furthermore, with the implemented feedback controller the deflection caused by the impact disturbance force 
$F_{d}(t)$ in Fig. 6 is compensated by the active force $F_{a}(t)$ forcing the column into its second bending deflection shape. In this case, the disturbance force is assumed to act at $x=L_{d}$. At this position, and without active stabilisation, mainly the first bending mode shape would be excited which makes the system very sensitive to buckle in its first buckling deflection shape. This position of $F_{d}$ was chosen for first investigations to test the worst case of possible disturbance forces causing buckling without active stabilisation.

\section{Experimental investigations}

To validate the presented concept for active stabilisation, a demonstrator has been built. Figure 9 shows the demonstrator active column system with different detail. First, the entire test rig with all instrumentation is shown, Fig. 9a. A strain gauge amplifier delivers signals from the strain gauge sensors to detect initial buckling. Those signals are filtered by an analogous low pass filter. The control is carried out with a real-time control system. Piezo amplifiers are used to drive the piezoelectric stack actuators with the control signals to stabilise the column. The measurement notebook is used to implement the controller on the real-time system.

Figure $9 \mathrm{~b}$ shows the demonstrator with two piezoelectric stack actuators which are oriented in opposite directions to apply the active force in both, positive and negative z-direction for buckling control, Fig. 6 . The system's boundary conditions are pinned-fixed and a mass acts as axial load just below the critical buckling load. The pendulum is used to apply a disturbance force $F_{d}=0.15 \mathrm{~N}$ with reproducible magnitude due to the pendulum guide limiting the pendulum's maximum deflection before releasing it. Strain gauge sensors measure the column's strain in real time to detect any deflection of the column and activate the piezoelectric stack actuators by a simple feedback controller to apply the stabilising active force $F_{a}$. As the actuators can only expand but not contract, they are prestressed electrically by an offset of half of their maximum voltage. The part attached to the piezoelectric actuators being in contact with the column is rounded in one dimension to apply a horizontal line force onto the column from both opposite sides, Fig. 9c bottom. Figure 9c top shows the pinned support and the limit stop to prevent the mass from falling down and the column from buckling plastically.

Experiments have been carried out for this stabilisation concept using a PID feedback control. The one-sided system could be stabilised for a $15 \%$ increased load and a disturbance of $F_{d}=0.15 \mathrm{~N}$ acting at the same time [12]. Using the same approach on the improved, two-sided system in Fig. 9b, an increase of $20 \%$ above the critical buckling load was achieved experimentally for the same location of the disturbance force. Further investigations are planned to improve the performance of the described system by means of a model based control approach with a closed loop state observer. To control higher buckling modes and, hence, bear higher axial loads, faster controllers are needed, as buckling time decreases with increasing axial load.

\section{Conclusions}

Uncertainty in stability and buckling due to unforeseen overloads may be controlled by active stabilisation. By implementing a controller to the column critical to buckling, the probability of the column's collapse is reduced. In this paper, existing concepts and a proposed new concept of active buckling control in simple column structures are discussed. The new concept is based on forcing the column into its second bending deflection shape by active control forces, applied near the column's fixed end by piezoelectric stack actuators. A feedback controller keeps the column in its second bending deflection shape to stabilise the column.

The main advantages of this approach are, firstly, the possibility to control not only buckling of a undisturbed system but also a buckling sensitive system with external disturbances and thus control uncertainty during operation. Secondly, the actuation mechanism allows to leave the major part of the column's surface unchanged and without additionally attached actuators to enable the correct operation of a functional surface.

In future work, a model based control algorithm for active buckling control will be designed first numerically for the shown system. In continuation, the control algorithm will be implemented and validated on the presented real test rig. 


\section{Acknowledgments}

The authors would like to thank the German Research Foundation (DFG) for funding this project within the Collaborative Research Centre (SFB) 805.

\section{References}

[1] H. Hanselka and R. Platz, Ansätze und Maßnahmen zur Beherrschung von Unsicherheit in lasttragenden Systemen des Maschinenbaus Controlling uncertainties in load-carrying systems, Konstruktion, 6, Springer VDI-Verlag, 2010, pp. 55-62,

[2] D. Gross, W. Hauger, J. Schröder and W. Wall, Technische Mechanik Band 2, Springer, 2007.

[3] S.P. Timoshenko and J.M. Gere, Theory of Elastic Stability, McGraw-Hill Book Company Inc., 1961.

[4] ISO/TS 13725, Hydraulic fluid power - Cylinders - Method for determining the buckling load, 2001.

[5] A.A. Berlin, Towards Intelligent Structures: Active Control of Buckling, Ph.D. Thesis, Dept. of Electrical Eng. and Computer Science, Massachusetts Institute of Technology, 1994.

[6] J.G. Chase and M. Yim, Optimal Stabilization of Column Buckling, Journal of Engineering Mechanics 125(9) ASCE (1999), 987-993.

[7] Q. Wang and V.K. Varadan, Transition of the Buckling Load of Beams by the Use of Piezoelectric Layers, Smart Materials and Structures 12(5) (2003), 696-702.

[8] Q.S. Wang, Active Buckling Control of Beams Using Piezoelectric Actuators and Strain Gauge Sensors, Smart Materials and Structures 19(6) (2010), 065022.

[9] T. Meressi and B. Paden, Buckling control of a flexible beam using piezoelectric actuators, Journal of Guidance, Control and Dynamics 16 ASME (1993), 977-980.

[10] S. Choi, J.J. Lee, D.C. Seo and S.W. Choi, The active buckling control of laminated composite beams with embedded shape memory alloy wires, Composite Structures 47, 1999, pp. 679-686.

[11] R.P. Jefferis, Feedback control of the buckling instability in an axially compressed thin elastic beam, Ph.D. Thesis, Dept. of Electrical Eng., University of Pennsylvania, 1968.

[12] G.C. Enss, R. Platz and H. Hanselka, An approach to control the stability in an active column by one single piezoelectric stack actuator, ISMA2010 24th International Conference on Noise and Vibration Engineering, 20-22 Sept 2010, Leuven/Belgium, 2010, pp. 535-546. 

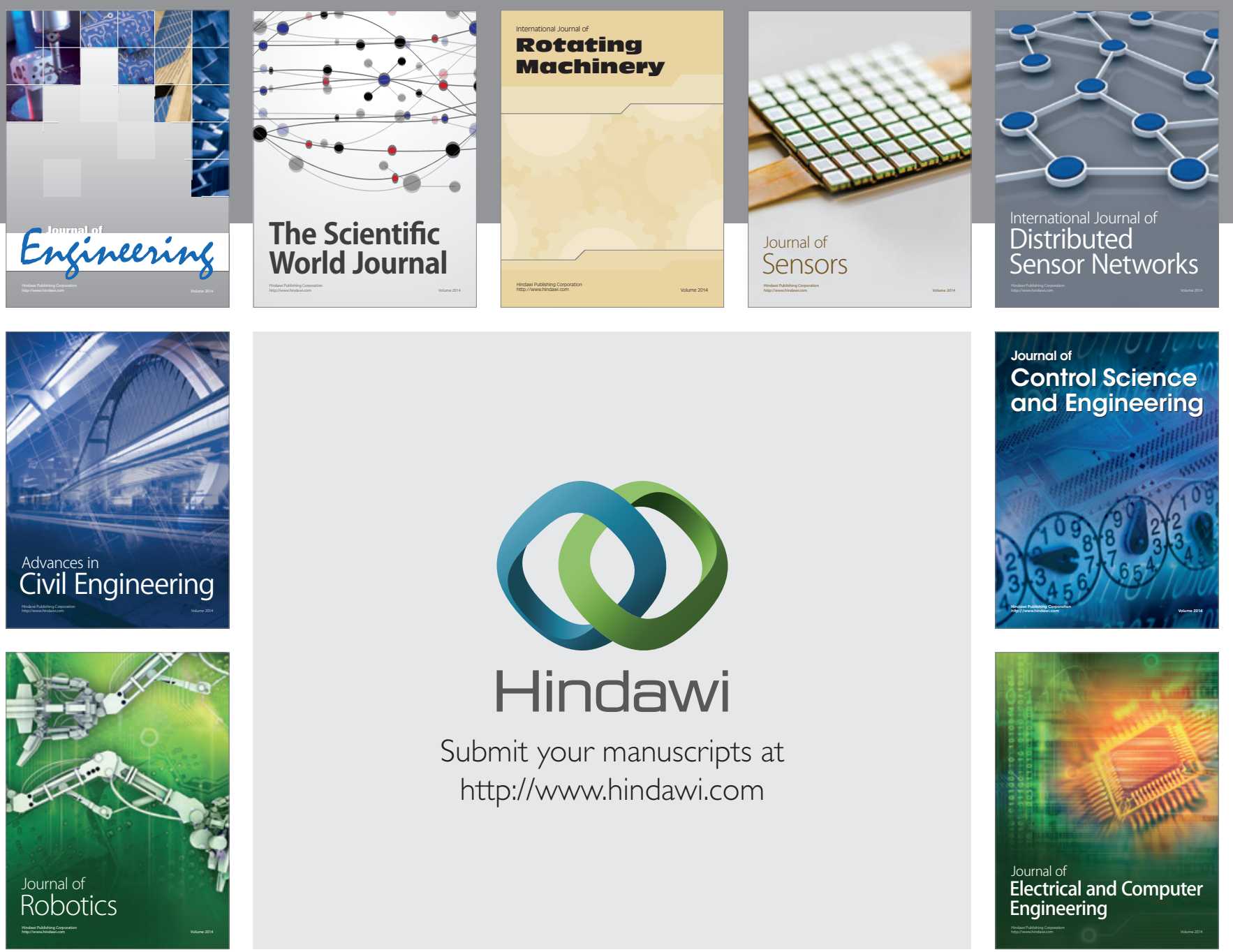

Submit your manuscripts at

http://www.hindawi.com
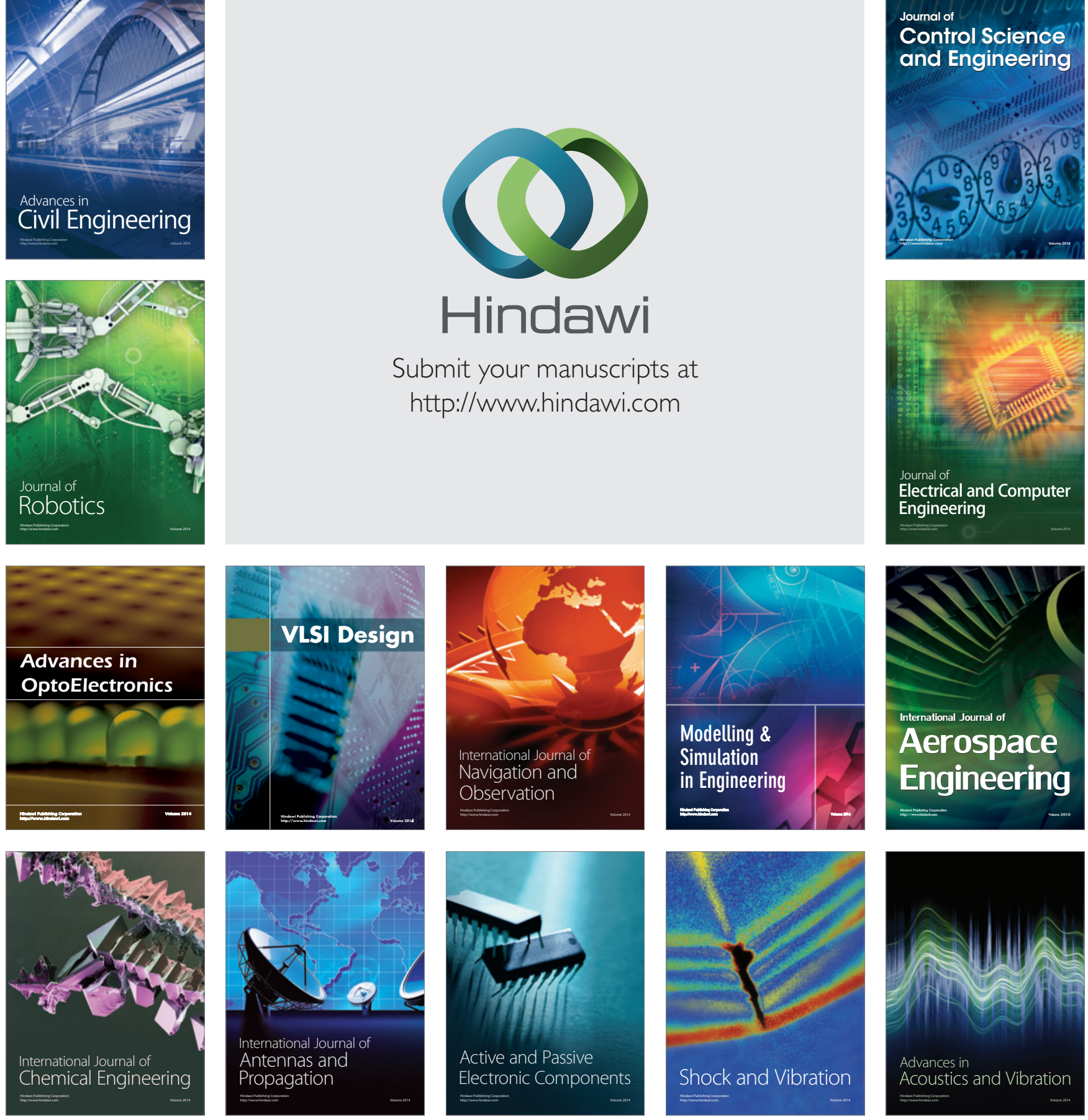